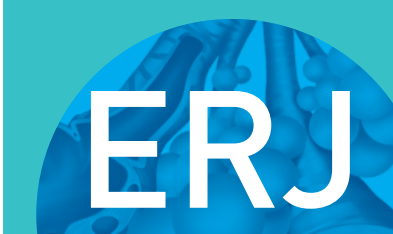

open research
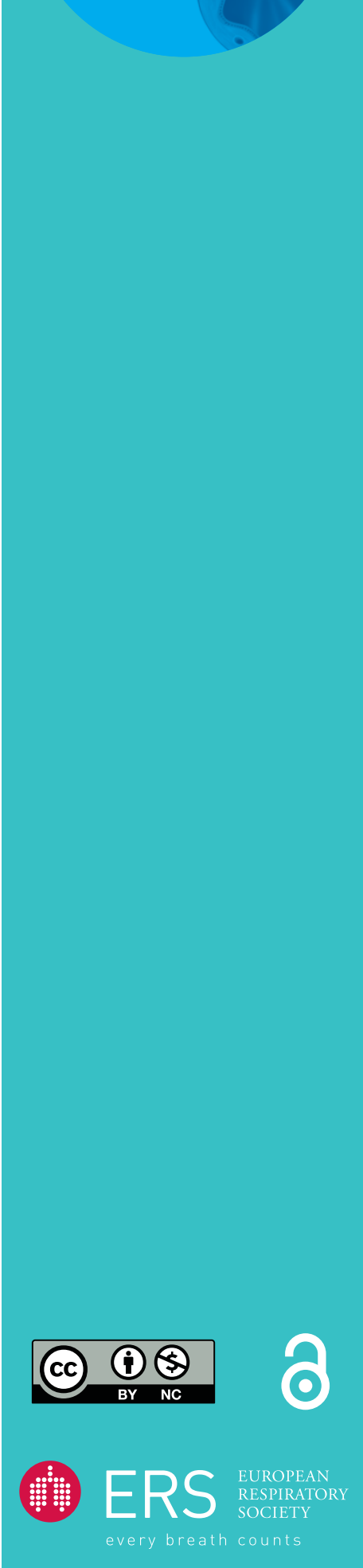

\section{EBUS-TBNA in the rapid microbiological diagnosis of drug-resistant mediastinal tuberculous lymphadenopathy}

\author{
Prashant N. Chhajed ${ }^{1,2}$, Preyas J. Vaidya ${ }^{1,2}$, Neha P. Mandovra ${ }^{1,2}$, \\ Vinod B. Chavhan ${ }^{1,2}$, Tejashree T. Lele ${ }^{1,2}$, Rekha Nair ${ }^{3}$, Jörg D. Leuppi ${ }^{4}$ and \\ Avinandan Saha ${ }^{1}$
}

Affiliations: 'Dept of Respiratory Medicine, Fortis Hiranandani Hospital, Navi Mumbai, India. ${ }^{2}$ Institute of Pulmonology, Medical Research and Development, Mumbai, India. ${ }^{3}$ Dept of Microbiology, Fortis Hiranandani Hospital, Navi Mumbai, India. ${ }^{4}$ University Clinic of Medicine, Kantonsspital Baselland, Liestal, Switzerland.

Correspondence: Prashant N. Chhajed, Institute of Pulmonology, Medical Research and Development, A405, 4th Floor, Sangam, SV Road and Saibaba Road Junction, Santa Cruz (West), Mumbai 400054, India. E-mail: pchhajeddamail.com

ABSTRACT This study aimed to examine the use of endobronchial ultrasound-guided transbronchial needle aspiration (EBUS-TBNA) in the rapid diagnosis of mediastinal tuberculous lymphadenitis and drug-resistant mediastinal tuberculous lymphadenitis.

A diagnosis of TB was confirmed by a positive Xpert MTB/RIF test or Mycobacterium tuberculosis culture. Rifampicin-resistant TB (RR-TB) or multidrug-resistant TB (MDR-TB) was diagnosed upon the detection of rifampicin resistance by Xpert MTB/RIF or resistance to rifampicin and isoniazid by phenotypic drug susceptibility testing (DST).

Xpert MTB/RIF was positive in 43 of 56 patients (77\%) and TB culture was positive in 31 of 56 patients (55\%). Of these 56 patients, 25 (45\%) were Xpert MTB/RIF positive and TB culture negative, 13 (23\%) were Xpert MTB/RIF negative and TB culture positive, and $18(32 \%)$ were Xpert MTB/RIF positive and TB culture positive. 11 patients (20\%) had drug-resistant TB: seven with RR/MDR-TB, one with preextensively drug-resistant (XDR) TB, two with XDR-TB and one with isoniazid mono-resistance.

An Xpert MTB/RIF assay carried out on EBUS-TBNA specimens provides rapid diagnosis of TB. Xpert MTB/RIF testing appears to have additional and more rapid sensitivity compared with culture alone. Culture-based DST provides an additional exclusive yield and the full resistance profile in addition to or instead of rifampicin resistance.

@ERSpublications

EBUS-TBNA has value in mediastinal adenopathy, including drug-resistant TB. Combined Xpert MTB/RIF and TB culture testing on EBUS-TBNA specimens helps in diagnosing rifampicin resistance missed by Xpert MTB/RIF. http://bit.ly/2LT2OOD

Cite this article as: Chhajed PN, Vaidya PJ, Mandovra NP, et al. EBUS-TBNA in the rapid microbiological diagnosis of drug-resistant mediastinal tuberculous lymphadenopathy. ERJ Open Res 2019; 5: 00008-2019 [https://doi.org/10.1183/23120541.00008-2019].

Received: 9 Jan 2019 | Accepted after revision: 23 Sept 2019

Copyright $\odot E R S$ 2019. This article is open access and distributed under the terms of the Creative Commons Attribution Non-Commercial Licence 4.0. 


\section{Introduction}

India had the highest burden of multidrug-resistant tuberculosis (MDR-TB) globally in 2015 [1, 2]. Epidemiological models predict that the incidence of MDR-TB in India will increase from 3.9 cases per 100000 population in 2012 to 14.1 per 100000 population in 2032. Primary transmission may account for $85 \%$ of the incidence by 2032 , compared to about $15 \%$ in 2012 [3]. To pre-empt this scenario, TB control efforts must focus on prompt diagnosis and treatment of MDR-TB [4]. Both World Health Organization (WHO) and Indian Revised National Tuberculosis Control Programme guidance emphasise the need to isolate the Mycobacterium tuberculosis pathogen in clinical specimens to enable drug susceptibility testing (DST). Rifampicin-resistant tuberculosis (RR-TB) detected by the Xpert MTB/RIF assay is a surrogate for MDR-TB because it is accompanied by isoniazid resistance in $95 \%$ of cases. Therefore, MDR-TB treatment regimens are recommended for both RR-TB as determined by Xpert MTB/RIF as well as MDR-TB based on DST results $[5,6]$.

Tuberculous lymphadenitis (TBLA) comprises $20 \%-40 \%$ of extrapulmonary TB cases [7]. The mediastinal lymph nodes, especially the hilar, paratracheal and subcarinal nodes, are usually affected. Mediastinal TBLA presents a diagnostic challenge due to nonspecific clinical features and difficulties in sampling the affected lymph nodes for microbiological and pathological analysis [7]. TBLA is usually paucibacillary, adding to the problems of obtaining a microbiological diagnosis [8]. Often, in TB endemic countries such as India, patients with mediastinal lymphadenopathy with a clinical suspicion of TB are offered empiric antitubercular therapy (ATT), typically first-line ATT. However, over the last decade there has been an increase in the occurrence of drug-resistant $\mathrm{TB}$ in several $\mathrm{TB}$ endemic countries, including India. A diagnosis of drug-resistant TB can only be made based on microbiological testing of tissue samples. Furthermore, treatment regimens that have been individualised following DST have shown better outcomes in the treatment of drug-resistant TB compared to protocol-based fixed regimens [9]. Therefore, tissue sampling for microbiological testing is necessary.

Obtaining tissue samples from enlarged mediastinal lymph nodes is possible using computed tomography-guided biopsy, mediastinoscopy, video-assisted thoracoscopic surgery or real-time endobronchial ultrasound-guided transbronchial needle aspiration (EBUS-TBNA) [10-12]. EBUS-TBNA enables safe, minimally invasive sampling of the mediastinal and hilar lymph nodes for microbiological and pathological analyses. The diagnostic role of EBUS-TBNA in mediastinal TBLA has been examined [11, 13]. However, there are limited data on the utility of EBUS-TBNA in the diagnosis of drug-resistant TB.

The current study describes the use of EBUS-TBNA in the rapid diagnosis of mediastinal TBLA and drug-resistant mediastinal TBLA, and assesses the diagnostic yields of genotypic and phenotypic microbiological investigations carried out on EBUS-TBNA specimens in an area with a high incidence of TB.

\section{Methods}

This descriptive, cross-sectional, retrospective, observational study was carried out at a tertiary care centre in the Mumbai region, an MDR-TB endemic region. Consecutive adult patients over a period of 4 years who underwent EBUS-TBNA that led to a microbiological diagnosis of TB were included in the study. Follow-up of all patients was not possible because ours is a referral centre, with several patients referred to us for the EBUS-TBNA procedure. These patients returned to their referring care providers following the procedure.

Data were collected from hospital and laboratory databases, and included EBUS-TBNA procedure details with results of microbiological and pathological investigations carried out on the specimens, as well as available clinical details. Institutional ethics committee approval was obtained. Patient consent was waived owing to the retrospective nature of the study. All patient records were anonymised and de-identified.

First, sputum microscopy was carried out on samples from all patients undergoing EBUS-TBNA who could produce sputum. Patients were also evaluated for more accessible lymph nodes. EBUS-TBNA was advised when these failed to yield results. Standard pre-procedure investigations included haemogram, biochemical and coagulation parameters. All patients underwent high-resolution computed tomography of the chest with contrast enhancement prior to EBUS-TBNA to evaluate the lymph nodes. EBUS-TBNA was performed with nebulised and "spray-as-you-go" local anaesthesia using 1\% lignocaine, and conscious sedation using a combination of fentanyl and midazolam with or without propofol administered intravenously. A convex probe echo-bronchoscope (BF-UC160F/180FOL8; Olympus, Tokyo, Japan) was used. Lymph nodes were sampled with a 21-gauge Olympus ViziShot single-use aspiration needle [10, 11].

Rapid on-site evaluation was not available and all specimens were sent directly to the laboratory. Tissue cores in formaldehyde were sent for histopathological examination. Core biopsies and aspirates flushed in saline were sent for Xpert MTB/RIF assay on the GeneXpert platform (Cepheid Inc., Sunnyvale, CA, USA) 
and mycobacterial culture. Aspirates were immediately smeared on slides, fixed with $95 \%$ ethanol and sent for cytology.

Microscopy for acid-fast bacilli was performed by Ziehl-Neelsen staining. The Xpert MTB/RIF assay was carried out according to the manufacturer's instructions to rapidly detect rifampicin resistance. Mycobacterial culture was carried out using the BACTEC MGIT 960 automated liquid culture system (Becton, Dickinson and Company, Franklin Lakes, NJ, USA). Final DST was carried out for all cultured M. tuberculosis isolates phenotypically on the MGIT 960 platform using the modified proportion method. Susceptibility to rifampicin, isoniazid, ethambutol, pyrazinamide, kanamycin, capreomycin, moxifloxacin/ ofloxacin, para-amino salicylic acid, ethionamide and clofazimine was tested. The drug concentrations employed for testing were based on WHO and manufacturer's (Becton, Dickinson and Company) recommendations for DST on MGIT 960 [14, 15].

A diagnosis of TB was confirmed from EBUS-TBNA specimens by a positive Xpert MTB/RIF result or a positive $M$. tuberculosis culture. A diagnosis of RR-TB/MDR-TB was made upon the detection of rifampicin resistance by Xpert MTB/RIF or resistance to rifampicin and isoniazid by MGIT 960-based phenotypic DST [5].

Extensively drug-resistant tuberculosis (XDR-TB) was defined as MDR-TB with additional resistance to any fluoroquinolone and a second-line injectable drug such as kanamycin, amikacin or capreomycin, and preXDR-TB was defined as MDR-TB associated with resistance to fluoroquinolone or a second-line injectable, but not both [16-18].

Pathological findings were classified as previously described: grade I, epithelioid granulomatous reaction with caseation; grade II, epithelioid granulomatous reaction without caseation; grade III, non-granulomatous reaction with necrosis; grade IV, nonspecific inflammation; grade V, inadequate specimen $[13,19]$.

Descriptive statistics were used in terms of percentages, frequencies, minima and maxima. The time taken to diagnosis was the time period between the EBUS-TBNA procedure and the issuance of an Xpert MTB/ RIF and/or phenotypic DST report by the laboratory based on which anti-RR-TB/MDR-TB treatment could be initiated.

\section{Results}

During the study period of 4 years, 266 patients underwent EBUS-TBNA. 56 patients had TB confirmed by microbiological examination (Xpert MTB/RIF positive, TB culture positive or both) of EBUS-TBNA specimens: 31 (55\%) were male and $25(45 \%)$ female. The median age of patients in our study was 34 years (range 13-78 years). All were of Indian ethnicity and none had HIV co-infection. Of these 56 patients, results of the Xpert MTB/RIF assay were positive in 43 (77\%) and TB culture was positive in 31 (55\%); 25 (45\%) were Xpert MTB/RIF positive and TB culture negative, 13 (23\%) were Xpert MTB/RIF negative and TB culture positive, and 18 (32\%) were Xpert MTB/RIF positive and TB culture positive.

The microbiological yields of EBUS-TBNA by Xpert MTB/RIF assay and TB culture are shown in figure 1. Of the 13 patients who were Xpert MTB/RIF negative and TB culture positive, the subsequent DST revealed pan-sensitive TB in 11 patients, preXDR-TB in one patient and XDR-TB in one patient. Of the 36 patients who were Xpert MTB/RIF positive with rifampicin-sensitive TB, the TB culture was positive in 15 patients and DST revealed pan-sensitive TB in 12 patients, MDR-TB in one patient and monoresistance to isoniazid in one patient; DST could not be performed in one patient. Of the seven patients who were Xpert MTB/RIF positive with RR-TB, the TB culture was positive in three patients and DST revealed MDR-TB in one patient and XDR-TB in one patient; DST could not be performed in one patient. Therefore, in two patients who were TB culture positive, DST could not be performed owing to difficulties in reviving the culture isolates for DST purposes. These two patients were treated with ATT on the basis of Xpert MTB/RIF results and clinical correlation. None of the patients with drug resistance had a past history of TB.

The median days to diagnosis of drug-resistant TB from EBUS-TBNA specimens was 12 days, with a range of 1 day (for Xpert MTB/RIF) to 60 days (for culture-based DST). Xpert MTB/RIF reports were available in 1-3 days (median 1 day). Culture-based DSTs, where possible, were available in 21-60 days (median 30 days) counting from the day of first collection of the specimen.

48 patients (86\%) with microbiological evidence of TB showed granulomatous inflammation on histopathological examination (grades I and II), while the remaining eight patients showed nonspecific inflammation (grade IV) or anthracosis. Of the 11 patients with microbiologically confirmed drug-resistant TB, six showed grade I, four showed grade II and one showed grade IV pathological findings. Of the 25 patients who were Xpert MTB/RIF positive and TB culture negative, 22 (88\%) showed granulomatous 


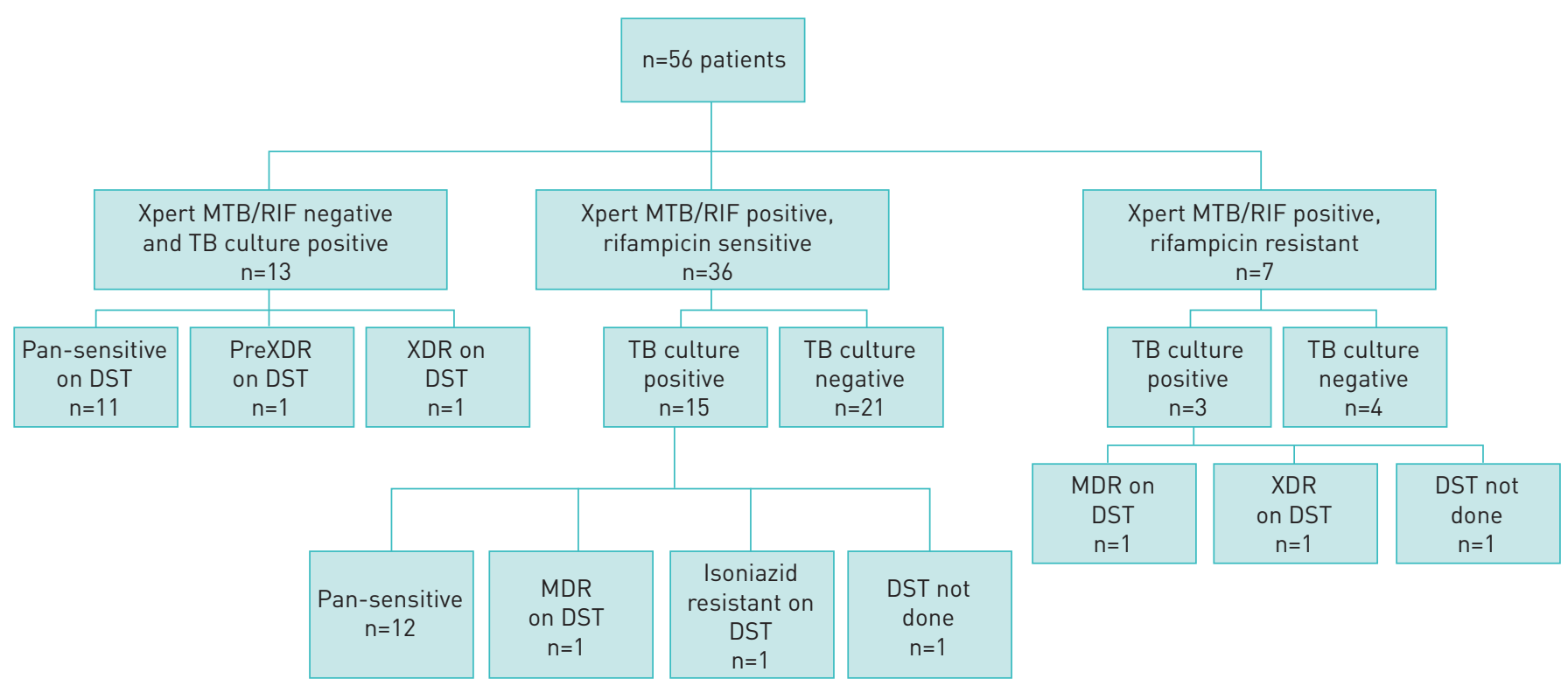

FIGURE 1 Xpert MTB/RIF assay, tuberculosis (TB) culture and drug susceptibility testing (DST) in the study population. MTB: Mycobacterium tuberculosis; RIF: rifampicin; XDR: extensively drug resistant; MDR: multidrug resistant.

inflammation on histopathology and three (12\%) showed chronic inflammatory changes. Furthermore, 24 of these 25 patients were treated for TB based on the clinical microbiological and histological correlation. Treatment information was not available for one patient.

\section{Discussion}

Mediastinal lymphadenitis is a common finding in pulmonary TB and may by itself account for up to $40 \%$ of extrapulmonary TB cases [7]. The diagnosis of intrathoracic TBLA traditionally relied on clinical and radiographic findings, as well as a therapeutic trial with anti-tubercular drugs. However, this can lead to redundant treatment, risk of adverse effects and a delay in the actual diagnosis and treatment. Microbiological and histopathological examination of the lymph nodes is indispensable for obtaining a definitive diagnosis, making sampling of the lymph nodes essential [7].

Mediastinoscopy was long considered the gold standard for sampling mediastinal lymph nodes, but it is invasive and requires general anaesthesia. Also, posterior subcarinal and hilar lymph nodes, which are commonly affected in TB, cannot be accessed. EBUS-TBNA enables sampling of the mediastinal lymph nodes under real-time ultrasonographic guidance, making it safe and minimally invasive, and facilitating adequate sampling [20]. It compares well with conventional TBNA and mediastinoscopy, with a sensitivity of $80 \%$ and specificity of $100 \%$ for the detection of TB by both microbiological and histopathological examinations [21].

The rate of positive cultures from EBUS-TBNA specimens is $20 \%-63 \%$. A recent meta-analysis reported a culture positivity rate of $54 \%$ and a smear positivity rate of $30 \%$ [22]. Another meta-analysis showed that the pooled sensitivity and specificity of EBUS-TBNA for the diagnosis of intrathoracic TB were 0.80 and 1.00 respectively [21]. In the current study, 25 patients were Xpert MTB/RIF positive but TB culture negative. Several previous studies have identified specimens that were Xpert MTB/RIF positive and TB culture negative $[23,24]$. High Xpert MTB/RIF positivity and TB culture negativity has been attributed to low viable counts or non-viable tubercle bacilli due to either the decontamination procedure or the nature of the caseous lesion in the lymph node tissue, which may have contained dead tubercle bacilli [25]. The low sensitivity of culture as compared to that of Xpert MTB/RIF has also been attributed to the paucibacillary nature of extrapulmonary specimens with uneven distribution of bacilli and formation of clumps [26]. Such cases (Xpert MTB/RIF positive and TB culture negative) are likely to be true positives as corroborated by the high specificity of Xpert MTB/RIF [23, 25, 27]. In the current subgroup of Xpert MTB/RIF-positive and TB culture-negative patients, 22 of 25 patients showed granulomatous inflammation and three patients showed chronic inflammatory changes on histopathology. This suggests that there was a high correlation of Xpert MTB/RIF positivity with histopathological findings. Because the current study setting is endemic for TB, patients were treated for TB based on Xpert MTB/RIF results, histopathological findings and clinical symptoms, despite having a negative TB culture. Similar approaches 
have previously been used with successful treatment outcomes [27]. The possibility of cross-contamination is least likely because the Xpert MTB/RIF assay is a closed system and is processed in accredited laboratories [23].

The Xpert MTB/RIF assay has an overall sensitivity of $88 \%$ and specificity of $99 \%$ for the detection of M. tuberculosis, and an overall sensitivity of $95 \%$ and specificity of $98 \%$ for the detection of rifampicin resistance [28]. The assay has been validated for extrapulmonary specimens in a high-prevalence endemic region like India, showing a sensitivity and specificity of $90.68 \%$ and $99.62 \%$ for the detection of M. tuberculosis, and sensitivity and specificity of $81.82 \%$ and $100 \%$ for the detection of rifampicin resistance [8]. The relatively low diagnostic yields of drug-resistant TB from culture and Xpert MTB/RIF in our study could be attributed to the paucibacillary nature of TBLA and the association between bacillary load in tissue and detection of rifampicin resistance [8]. Xpert MTB/RIF-negative and TB culture-positive samples were observed in $23 \%$ of patients. It has been postulated that the concentration of mycobacteria in the GeneXpert-negative samples is lower than the limit of detection of the Xpert MTB/ RIF assay, resulting in the Xpert MTB/RIF-negative but TB culture-positive results [23].

We encountered patients in whom culture-based DST, but not Xpert MTB/RIF, revealed rifampicin resistance (figure 1). The Xpert MTB/RIF assay uses five molecular probes complementary to the wild type $r p o B$ gene in the rifampicin-resistance-determining region (RRDR) of the $M$. tuberculosis genome. Rifampicin-resistance mutations that inhibit binding of these probes can be detected in up to 95\% of cases [8]. It is therefore possible to miss drug-resistance mutations in the remaining 5\%, which may either lie in the RRDR and only delay binding of the probes, or lie outside the RRDR altogether [29].

DHASMANA et al. [30] reported three cases of MDR-TB out of 88 cases with microbiologically confirmed mediastinal TBLA: two were identified by Xpert MTB/RIF and one by line probe assay. Thangakunam et al. [31] did not find any rifampicin resistance on Xpert MTB/RIF testing of EBUS-TBNA aspirates. In comparison, we found that $20 \%$ of patients (11 out of 56) with microbiologically proven TB in our cohort had drug-resistant TB. This observation can be attributed to various factors, e.g. the high prevalence of MDR-TB in the region where the current study was undertaken or referral bias [32]. The current study does highlight the value of EBUS-TBNA specimens in the diagnosis of TB as well as drug-resistant TB. However, the actual incidence of drug-resistant TB in our TB-endemic region in EBUS-TBNA specimens would need to be assessed prospectively in a well-defined patient cohort.

Aspirate samples obtained from lymph nodes that had necrotising granulomas yielded a microbiological diagnosis. Sun et al. [33] reported that necrosis was a risk factor associated with a better chance of culture positivity. The tubercle bacilli reside in the necrotic regions of necrotising granulomas and sampling of these regions is important to obtain a diagnosis of TB.

The median days to diagnosis of drug-resistant TB by Xpert MTB/RIF was 1 day (range 1-3 days), and by DST was 30 days (range 21-60 days, including DST). The delay of a day or more for Xpert MTB/RIF testing in some cases was because the specimens were sent to an offsite laboratory. However, our findings are comparable to the findings of NAVANi et al. [13] (median time to positive culture, excluding DST, 16 days, range 3-84 days; one centre in the study extended incubation beyond 6 weeks) and of DHASMANA et al. [30] (mean time to positive culture for microscopy positive cases, excluding DST, 13.3 days, range 6-19 days).

The limitations of the current study include, first, the lack of a single adequate gold standard against which either Xpert MTB/RIF or MGIT 960-based DST may be measured. Positive results from either Xpert MTB/RIF or MGIT 960 were used as a reference standard and, indeed, taken as an indication to initiate MDR-TB treatment regimens in our patients. Supporting this was the evidence from literature documenting the high specificity and positive predictive values of both tests $[15,33]$. Second, the study institute serves as a referral centre and several patients were transferred back to their referring physicians following EBUS-TBNA and consequently were not followed up. Therefore, the current study cohort is unable to provide the outcome information for these patients. Further analysis on a larger sample of patients with drug-resistant TB with follow-up of treatment outcome would be useful.

In conclusion, the current study highlights that EBUS-TBNA is a valuable tool in the diagnosis of mediastinal TBLA, including drug-resistant M. tuberculosis. The Xpert MTB/RIF assay carried out on EBUS-TBNA specimens provides a rapid diagnosis in a significant proportion of patients with TB. However, all EBUS-TBNA specimens should also be sent for culture-based DST, which provides a likely paucibacillary population missed by the molecular technique but also detects resistance to drugs other than rifampicin and rifampicin resistance that may be missed by Xpert MTB/RIF testing. Combined Xpert MTB/RIF and TB culture testing on EBUS-TBNA specimens is therefore a useful modality in the diagnosis of drug-resistant mediastinal TBLA. 
Support statement: This study was supported by the Institute of Pulmonology Medical Research and Development. Funding information for this article has been deposited with the Crossref Funder Registry.

Conflict of interest: None declared.

\section{References}

1 Dheda K, Gumbo T, Maartens G, et al. The epidemiology, pathogenesis, transmission, diagnosis, and management of multidrug-resistant, extensively drug-resistant, and incurable tuberculosis. Lancet Respir Med 2017; 5: 291-360.

2 World Health Organization. Global Tuberculosis Report 2016. Geneva, World Health Organization, 2016. http:// apps.who.int/iris/bitstream/10665/250441/1/9789241565394-eng.pdf?ua=1 Date last accessed: January 4, 2019.

3 Law S, Piatek AS, Vincent C, et al. Emergence of drug resistance in patients with tuberculosis cared for by the Indian health-care system: a dynamic modelling study. Lancet Public Health 2017; 2: e47-e55.

4 Suen SC, Bendavid E, Goldhaber-Fiebert JD. Disease control implications of India's changing multi-drug resistant tuberculosis epidemic. PLoS One 2014; 9: e89822.

5 World Health Organization. WHO Treatment Guidelines for Drug-Resistant Tuberculosis 2016 Update. Geneva, World Health Organization, 2016. www.who.int/tb/areas-of-work/drug-resistant-tb/MDRTBguidelines2016.pdf Date last accessed: January 4, 2019.

6 Central TB Division, Directorate General of Health Services: Revised National Tuberculosis Control Programme. Guidelines on Programmatic Management of Drug Resistant TB (PMDT) in India. New Delhi, Central TB Division, 2012 http://tbcindia.gov.in/WriteReadData/1892s/8320929355Guidelines Date last accessed: January 4, 2019.

$7 \quad$ Gupta PR. Difficulties in managing lymph node tuberculosis. Lung India 2004; 21: 50.

8 Singh UB, Pandey P, Mehta G, et al. Genotypic, phenotypic and clinical validation of GeneXpert in extra-pulmonary and pulmonary tuberculosis in India. PLoS One 2016; 11: e0149258.

9 Saha A, Vaidya PJ, Chavhan VB, et al. Factors affecting outcomes of individualised treatment for drug resistant tuberculosis in an endemic region. Indian J Tuberc 2019; 66: 240-246.

10 Yasufuku K, Chiyo M, Sekine $\mathrm{Y}$, et al. Real-time endobronchial ultrasound-guided transbronchial needle aspiration of mediastinal and hilar lymph nodes. Chest 2004; 126: 122-128.

11 Chhajed PN, Kate AH, Vaidya PJ. Endobronchial ultrasound-guided transbronchial needle aspiration: the standard of care for evaluation of mediastinal and hilar lymphadenopathy. J Cancer Res Ther 2013; 9: 549-551.

12 Bilaceroglu S, Chhajed P. Transbronchial needle aspiration: a diagnostic tool in routine bronchoscopy. J Assoc Physicians India 2005; 53: 797-802.

13 Navani N, Molyneaux PL, Breen RA, et al. Utility of endobronchial ultrasound-guided transbronchial needle aspiration in patients with tuberculous intrathoracic lymphadenopathy: a multicentre study. Thorax 2011; 66: 889-893.

14 World Health Organization. Policy Guidance on Drug-Susceptibility Testing of Second-Line Antituberculosis Drugs. Geneva, World Health Organization, 2008. http://apps.who.int/iris/bitstream/10665/70500/1/ WHO_HTM_TB_2008.392_eng.pdf Date last accessed: January 4, 2019.

15 van Ingen J, Simons S, de Zwaan R, et al. Comparative study on genotypic and phenotypic second-line drug resistance testing of Mycobacterium tuberculosis complex isolates. J Clin Microbiol 2010; 48: 2749-2753.

16 Orenstein EW, Basu S, Shah NS, et al. Treatment outcomes among patients with multidrug-resistant tuberculosis: systematic review and meta-analysis. Lancet Infect Dis 2009; 9: 153-161.

17 Mitnick CD, Shin SS, Seung KJ, et al. Comprehensive treatment of extensively drug-resistant tuberculosis. $N$ Engl J Med 2008; 359: 563-574.

18 Jacobson KR, Tierney DB, Jeon CY, et al. Treatment outcomes among patients with extensively drug-resistant tuberculosis: systematic review and meta-analysis. Clin Infect Dis 2010; 51: 6-14.

19 Bezabih M, Mariam DW, Selassie SG. Fine needle aspiration cytology of suspected tuberculous lymphadenitis. Cytopathology 2002; 13: 284-290.

20 Vaidya PJ, Munavvar M, Leuppi JD, et al. Endobronchial ultrasound-guided transbronchial needle aspiration: safe as it sounds. Respirology 2017; 22: 1093-1101.

21 Ye W, Zhang R, Xu X, et al. Diagnostic efficacy and safety of endobronchial ultrasound-guided transbronchial needle aspiration in intrathoracic tuberculosis: a meta-analysis. J Ultrasound Med 2015; 34: 1645-1650.

$22 \mathrm{Li} \mathrm{W}$, Zhang $\mathrm{T}$, Chen $\mathrm{Y}$, et al. Diagnostic value of convex probe endobronchial ultrasound-guided transbronchial needle aspiration in mediastinal tuberculous lymphadenitis: a systematic review and meta-analysis. Med Sci Monit 2015; 21: 2064-2072.

23 Tadesse M, Abebe G, Abdissa K, et al. GeneXpert MTB/RIF assay for the diagnosis of tuberculous lymphadenitis on concentrated fine needle aspirates in high tuberculosis burden settings. PLoS One 2015; 10: e0137471.

24 Iram S, Zeenat A, Hussain S, et al. Rapid diagnosis of tuberculosis using Xpert MTB/RIF assay - report from a developing country. Pak J Med Sci 2015; 31: 105-110.

25 Hillemann D, Rüsch-Gerdes S, Boehme C, et al. Rapid molecular detection of extrapulmonary tuberculosis by the automated GeneXpert MTB/RIF system. J Clin Microbiol 2011; 49: 1202-1205.

26 Helb D, Jones M, Story E, et al. Rapid detection of Mycobacterium tuberculosis and rifampin resistance by use of on-demand, near-patient technology. J Clin Microbiol 2010; 48: 229-237.

27 Eom JS, Mok JH, Lee MK, et al. Efficacy of TB-PCR using EBUS-TBNA samples in patients with intrathoracic granulomatous lymphadenopathy. BMC Pulm Med 2015; 15: 166.

28 World Health Organization. Xpert MTB/RIF Implementation Manual Technical and Operational "How-To": Practical Considerations. Geneva, World Health Organization, 2014. http://apps.who.int/iris/bitstream/10665/ 112469/1/9789241506700_eng.pdf Date last accessed: January 4, 2019.

29 Blakemore R, Story E, Helb D, et al. Evaluation of the analytical performance of the Xpert MTB/RIF assay. J Clin Microbiol 2010; 48: 2495-2501.

30 Dhasmana DJ, Ross C, Bradley CJ, et al. Performance of Xpert MTB/RIF in the diagnosis of tuberculous mediastinal lymphadenopathy by endobronchial ultrasound. Ann Am Thorac Soc 2014; 11: 392-396.

31 Thangakunam B, Isaac BT, Christopher DJ. Endobronchial ultrasound experience in a high tuberculosis prevalence setting. Indian J Tuberc 2017; 64: 196-200. 
32 Dalal A, Pawaskar A, Das M, et al. Resistance patterns among multidrug-resistant tuberculosis patients in Greater Metropolitan Mumbai: trends over time. PLoS One 2015; 10: e0116798.

33 Sun J, Teng J, Yang H, et al. Endobronchial ultrasound-guided transbronchial needle aspiration in diagnosing intrathoracic tuberculosis. Ann Thorac Surg 2013; 96: 2021-2027. 